\title{
Autophagy: eat thyself, sustain thyself
}

\author{
Vivien Marx
}

\section{A growing research community studies autophagy - a process of cellular recycling and maintenance-but there are some hot-button methodological issues.}

A student with his seeing-eye dog approached cell biologist Dan Klionsky of the University of Michigan after one of his talks. He thanked Klionsky for including the musical score Macromusophagy in his presentation, because it had allowed the young scientist to 'hear' the phases of autophagy, a process of cellular housekeeping in which cells maintain themselves by degrading faulty organelles or accumulated proteins and recycling metabolites. Klionsky had invited composer Wendy Wan-Ki Lee to create that piece.

Macromusophagy is also part of Klionsky's collaboration with an illustrator and dancers. Klionsky gave choreographer Peter Sparling poetic license to render the complex phases of autophagy in the language of modern dance. The piece was "tremendous fun" to develop and perform, says Klionsky.

When he is not integrating art and science, Klionsky studies autophagy in yeast. $\mathrm{He}$ and 2,460 coauthors have finalized a paper now in press in Autophagy, of which Klionsky is the editor, describing updated guidelines for the use and interpretation of assays to monitor autophagy ${ }^{1}$.

\section{Growing popularity}

For the upcoming guideline paper, Klionsky winnowed through a mountain of 16,000 papers related to autophagy, many of them on systems other than yeast, and many introducing new terms or methods. The last guidelines, published in 2012, had only half as many authors. Many factors have contributed to this growth.

Autophagy is a process of cellular housekeeping in which dysfunctional organelles, aggregated macromolecules, misfolded proteins or pathogens are removed and degraded. Some of the metabolites are reused. Autophagy is also dynamic-it helps cells

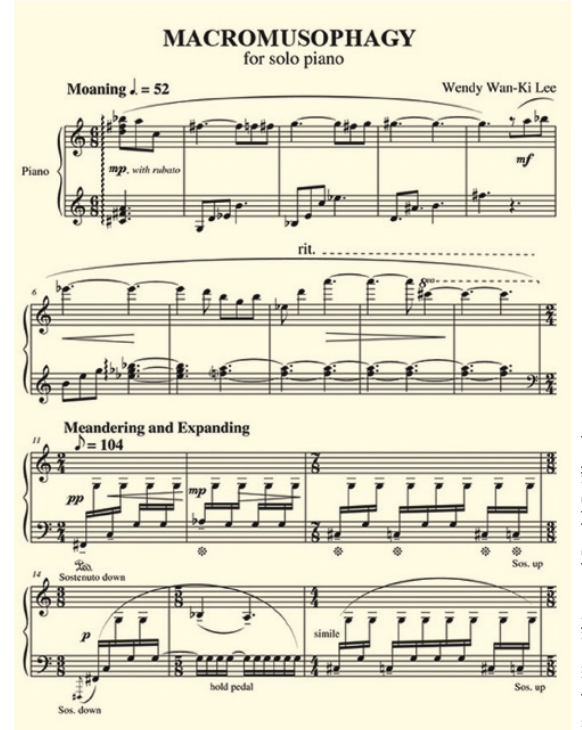

Macromusophagy is a musical score developed at the Chinese University of Hong Kong and the University of Michigan.

react to environmental changes, including extreme situations such as starvation or deprivation of amino acids.

Researchers increasingly see that autophagy has a role in many biological processes such as development, aging and immunity. Its misregulation appears to be a culprit in conditions as diverse as inflammatory diseases, cancer and neurodegeneration. The spectrum of findings is perhaps not surprising, says Klionsky. Autophagy is now appreciated as a fundamental cellular process that can affect physiology in many ways.

What intrigues Goethe University researcher Ivan Dikic is the multitude and scope of 'good' autophagic processes, their multiple roles in physiology and the fact that there can also be an "unexpected shift toward pathological autophagy." Autophagy is a strategy for cellular self-fortification, but it is also a balance: too much or too little autophagy can be harmful to the cell. The field has grown to include scientists of "all flavors," as cancer biologist Kay Macleod of the University of Chicago phrases it. She studies the role of autophagy in cancer and is developing ways to track the autophagic process in vivo in mice.

To study autophagy, researchers apply a number of methods such as electron and fluorescence microscopy, western blotting to track the processing of certain marker proteins, and flow cytometry, but, says Macleod, "we all recognize that our ability to monitor and measure autophagy is not as good as it should be." She is optimistic that the surging community will develop and validate better tools to characterize the process qualitatively and quantitatively. In the meantime she is encountering a number of what she calls "hot-button issues." One of them is that researchers base their claims on findings from one method, instead of multiple methods.

Klionsky, too, identifies methodological issues, pointing out that static measurements should not be used to draw conclusions about autophagic flux (the entire process of autophagy, from autophagosome biogenesis through to degradation in the lysosome). Such studies necessitate multiple measurements at different time points and the use of inhibitors to block the last stage of the process, for comparison.

He draws an analogy to a city block on which garbage has amassed. One may conclude that the area's inhabitants have been producing more garbage than is typical, but there might also be a strike. The outcome looks the same, but the causes are completely different. Similarly, cellular autophagy might seem upregulated under certain conditions, but there might also be a 


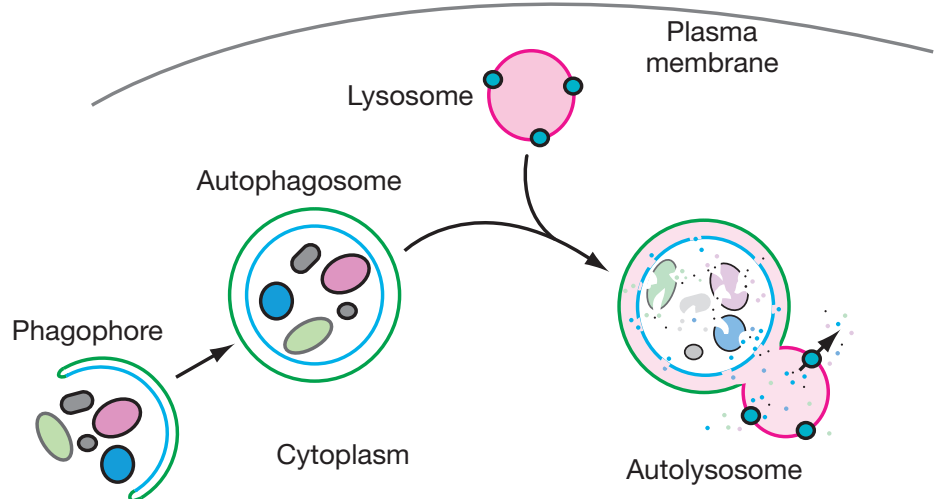

Autophagy is a process of cellular housekeeping, during which dysfunctional organelles, misfolded proteins or pathogens are removed and degraded. Some of the metabolites are then reused.

block in one of the last stages of the process. "Looking at one time point will not tell the answer," says Klionsky.

Attentiveness to such issues can enable comparison of data and analyses. Especially in a growing field, says Macleod, having comparable results from one lab to the next is quite crucial. As Klionsky explains, the guidelines aim to facilitate the review of manuscripts not just at his journal but, he hopes, at others as well. They can guide the community not as rigid rules but as recommendations.

\section{Identifying structures}

Autophagosomes are double-membraned structures in which cellular components are sequestered and readied for degradation. But they are not always obvious to identify, Klionsky says.

Macleod regularly sees researchers overinterpreting electron microscopy (EM) images and also forgetting that the images are a view of one section in one crowded location of the cell. The cytoplasm has plenty of complex structures, she says. Interpreting EM images to identify autophagic structures with certainty takes much expertise $^{2}$.

EM is often called the gold standard for identifying structures involved in the autophagic process and confirming findings from fluorescence microscopy, says Klionsky. But "it is by its very nature static," he says, whereas autophagy is a dynamic process. The autophagosome fuses with a lysosome, creating an autolysosome, where degradation occurs. EM can be used for autophagic flux measurements only if scientists image at multiple time points. Many researchers also do not perform immunoEM, says Klionsky. This is the preferable method, in his view, as it can be used to tell whether the structure in a micrograph is lined with a protein such as LC3 (microtubule-associated protein 1 with light chain 3 ).

\section{Reliance on LC3}

Autophagy researchers are reliant on measurements of LC3, its isoforms and processed variants thereof, says Macleod. Cytosolic LC3 is cleaved to LC3-I and conjugated to phosphatidylethanolamine, which is recruited to the pre-autophagosomal membrane as LC3-II. The different forms of LC3 can be followed by western blotting; for instance, one experimental approach looks at conversion from LC3-I to LC3-II. The protein can also be tagged with fluorophores, enabling a researcher to find autophagosomes and to measure autophagic flux with fluorescence microscopy.

Scientists in this field have long and rather turbulently discussed the interpretation of western blots related to LC3, says Macleod ${ }^{3}$. An important issue too often forgotten is that LC3 levels can change for many reasons. When autophagy is inhibited or modulated, the cell can feed back and upregulate LC3 at the transcriptional level. In fact, a new focus in the field is addressing how transcriptional regulators of autophagy and of lysosomal biogenesis contribute to the overall control of autophagy and responses to nutrient stress, she says. The nuclear levels of these transcription factors help scientists understand how autophagy can be deregulated in diseases such as cancer.

Fluorescent proteins such as GFP-LC3 and mCherry-EGFP-LC3 help scientists track LC3 with fluorescence microscopy. Klionsky thinks highly in particular of the dual-tagged proteins for following LC3 in mammalian cells. Dual tags let a researcher follow autophagic flux because GFP fluorescence is quenched in the acidic lysosome. Tagged autophagosomes in the cytosol appear yellow, but after they fuse with the lysosome, the green fluorescence is quenched and an experimenter sees only red. Comparing the number of yellow to red dots gives some idea of flux, he says.

But these proteins need to be overexpressed in the cell, which can itself induce autophagy, says Macleod. "You're actually messing with the thing you are trying to measure, so that's the problem there."

In instances when LC3 cannot be measured in a reliable fashion, as can be the case

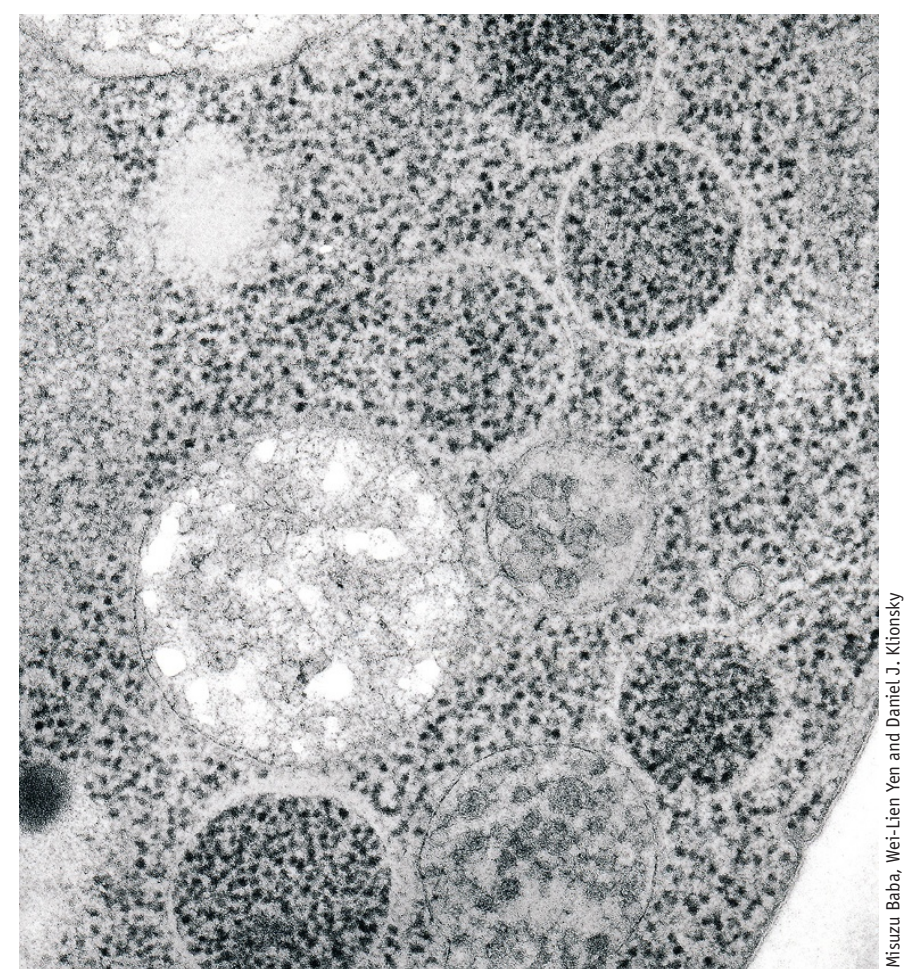

With electron microscopy, researchers can study autophagy. But it is not always easy to identify autophagosomes, where cellular components are sequestered prior to degradation. 
in tumor sections, Macleod sees scientists measuring levels of the protein p62, which is turned over in autophagy. When autophagy is inhibited, p62 levels rise, so accumulated p62 is used as a proxy in autophagy measurements. But what is often underappreciated, she says, is that p62 is regulated by many pathways, including NF- $\kappa \mathrm{B}$ and tumor necrosis factor $-\alpha$ signaling. That means a change in p62 levels is not a surefire indication that a sample is showing autophagy defects or increases. "My feeling on that is sometimes we have no choice, but it's clearly a measurement that's open to caveats," she says. Using levels of processed LC3, LC3-II and p62 can make researchers more confident about their data.

Dikic also points to the challenges associated with overexpression when tagging proteins with fluorophores. He uses multiple methods to be sure about his data: biochemical assays that assess LC3 processing and p62 levels, fluorescence microscopy using double-tagged GFP-mCherry fusion proteins to assess LC3 levels, and EM. One may also use fluorescence-activated cell sorting to look for the accumulation of processed, fluorophore-tagged LC3. "I like the doubletagged fusion proteins, as they indicate the final delivery to the lysosome," he says.

\section{Selective markers}

Autophagy can be a quite selective process. For example, only certain organelles such as ribosomes and peroxisomes, the endoplasmic reticulum (ER) and part of the nuclear membrane may be autophagocytosed. Macleod is particularly interested in the selective turnover of mitochondria in autophagy (mitophagy) and is exploring the signals that lead broken mitochondria to be selected and then degraded. She is also exploring the roles mitochondria play in cancer cells and metastasis.

Macleod's experimental challenge has been that she has needed to stain separately for autophagosomes and for mitochondria and then look for overlap in the signals, "which, when you're doing that under the microscope, is somewhat subjective," she says. "You're looking for yellow in a sea of green and red." Although software such as ImageJ can help with image analysis, she has begun exploring another approach using mito-monomeric Keima (mt-mKeima), a fluorescent proteinbased sensor based on mKeima, both of which were developed at RIKEN ${ }^{4,5}$.

When the mitochondria are in the cytosol, the sensor emits green fluorescence, but

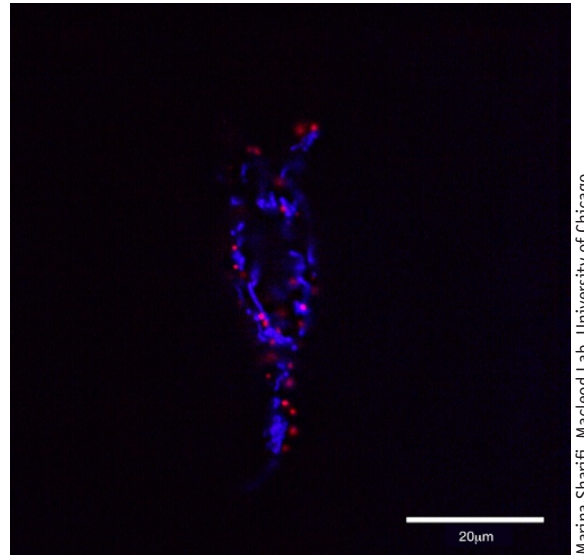

In contrast, when mitophagy in particular was inhibited in mouse models, tumors metastasized more rapidly. Such findings, says Macleod, show why scientists must be circumspect in their interpretation of studies that involve inhibition of general autophagy. They also emphasize how important it is to inhibit different types of autophagy, such as mitophagy, in isolation. If fluorophores could be targeted to organelles and also not interfere with the autophagy process, they would provide a way to monitor autophagy geographically within a cell, within tissue, in an organism and over time. But until those tools are available and can be validated, researchers need to be frank about their findings, says Macleod.

Scientists working with mammalian cells still lack what Klionsky and other yeast researchers have at their disposal: a truly quantitative assay. In yeast, there is nonselective and selective autophagy, as well as the unique cytoplasm-to-vacuole targeting (Cvt) pathway, which is selective as well as biosynthetic, says Klionsky. One can measure autophagy in yeast by monitoring the activity of a modified phosphatase, Pho8, that is typically present in the cytosol in an inactive form but moves into the vacuole during autophagy. Autophagy activates that enzyme, says Klionsky, which "gives us a nice enzymatic readout."

Pho8 is a vacuolar enzyme that normally goes to the vacuole through the secretory pathway. Klionsky and his team modify Pho8 to keep it in the cytosol-by removing its signal sequence-and can thus use it to monitor nonselective autophagy. They used Pho8 for this purpose because, he says, there are basically no great enzymatic assays for monitoring other vacuolar enzymes, such as proteases.

Klionsky's lab previously characterized aminopeptidase I, a vacuolar protease that moves to the vacuole through the Cvt pathway or autophagy ${ }^{6}$. Aminopeptidase I can be used to follow the normal secretory pathway-independent itinerary to the vacuole, with gel electrophoresis used as the readout. When Klionsky's lab published this work, many scientists approached him for plasmids so they could try this approach in their systems. But the method cannot be readily transferred to other organisms, given that it requires certain receptors and a scaffold protein to be present. And, says Klionsky, there just may be no Cvt pathway in mammalian cells, although there are certainly other types of selective autophagy. 


\section{Inhibiting, inducing}

In their experiments, scientists use small molecules that act as both inhibitors and inducers of autophagy. Rapamycin can induce autophagy, and, for example, 3-methyladenine, bafilomycin and chloroquine inhibit it. As Macleod explains, many experimenters use these reagents for too long and at too high a concentration, which leads to results that vary from one lab to the next.

The interpretation challenge is magnified by the effect the inducer or inhibitor has on protein levels, says Klionsky. LC3 levels might increase after a compound is added because autophagy is induced. But the compound might also affect the later stages of autophagy, which will also lead protein levels

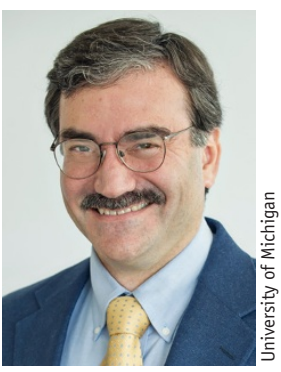

to rise, but for the opposite reason; this risks experimenters drawing the wrong conclusion from their experiment.

What makes these misinterpretations especially treacherous is that

It is better to not draw conclusions about autophagic flux from static measurements, says Dan Klionsky. drug candidate might be thought to induce autophagy when in fact it blocks autophagy.

Labs are searching for ways to monitor autophagy in vivo, which could lead to the use of imaging methods to study drug candidates. There is much interest in modulating autophagy for clinical purposes, says Klionsky, but the lack of approaches to determine whether or to what extent a particular drug has affected autophagy makes it hard to modulate the process effectively. In neurodegenerative conditions, for example, compromised autophagy is considered a contributor to neuronal cell death. That makes it tempting to explore how one might upregulate autophagy in a patient. But before that can happen, basic biology research on autophagy is needed. An approach cannot simply involve maximal induction of autophagy, because, Klionsky says, "you can't simply turn it on, period, because that can kill a cell."

\section{Commercial assays}

Companies offer many autophagy-related products. Many companies propose that scientists contact them even when they are still in the experimental design stage as well as when experiments are under way. This can help labs match the right reagents to their experiment.

Jack Coleman, who directs biochemistry at Enzo Life Sciences, explains that his company sells a proprietary dye, an amphiphilic tracer that stains lysosomes minimally while maximizing the fluorescence of autophagosomes, as part of a kit called Cyto-ID. The kit can be used to monitor autophagy in live cells. When used with inhibitors, the dye's specificity helps researchers distinguish the inhibition of lysosomal fusion from an increase in autophagic flux, he says. Other dyes too often show both lysosome and autophagosome, and, he says, his company's dye stays put, whereas other dyes tend to disappear from the assay when certain inhibitors are used.

The market has a broad selection of antibodies to LC3, but it also seems that many of them fail. Enzo application scientist Morgan Mathieu sees researchers sometimes struggling to distinguish between different forms of LC3. These isoforms can make it hard to interpret western blots, and researchers thus risk drawing incorrect conclusions from the obtained data. For experimental results to be informative, says Mathieu, the details matter. Researchers need to prepare the sample in the right lysis buffer, they must use the right controls, and they must have the appropriate membranes for western blotting, because of the lipophilic nature of LC3-II. Any of these factors can contribute to difficulties in analyzing LC3 by western blotting.

EMD Millipore scientist Chandra Mohan also notes that antibodies can react to both LC3-I and LC3-II. Researchers need to conduct a sufficient number of experiments so they can study the western blot bands, see the trends in their samples and learn which levels are rising.

LC3-I is mainly in the cytosol, and its predominance means the cell is not yet fully committed to autophagy. LC3-II binds to the autophagosomal membrane and is plentiful when the cell is firmly committed to autophagy, says Mohan. EMD Millipore sells a reagent kit that is tailored to Muse, the company's flow cytometer. Scientists can label cells for the LC3 marker and then sort and count the cells to measure autophagy. 
Mohan sees a growing number of researchers approaching his company for reagents and kits related to autophagy, given the renaissance the field is experiencing in both stem cell biology and cancer research. In stem cells, autophagy is involved in maintaining quiescence, and it has a role in cellular remodeling during differentiation, he says. Cancer cells activate autophagy in response to cellular stress and increased metabolic demands for proliferation, he says. And the inhibition of autophagy can restore the chemosensitivity of cancer cells.

When it comes to inducers and inhibitors, Enzo scientist Jianjun Bao suggests that customers use low dosages and low incubation times, which will help them avoid the off-target effects that these compounds can have. The company tests inducers and inhibitors in different cell types and under different conditions to discover the minimal dosage needed, she says.

Another recommendation is to measure cell viability and not just autophagy, as off-target effects can affect cell viability, says Bao. An experimenter might trigger autophagy as well as apoptosis or other signaling pathways inside the cell.

Mohan says he encounters researchers who complain that they do not see inhibition when using a millimolar quantity of an inhibitor with a half-maximal inhibitory concentration $\left(\mathrm{IC}_{50}\right)$ in the nanomolar range. It is a common belief, says Mohan, that using a larger amount of an inducer or an inhibitor will increase its effect. He finds that he must repeatedly lay that misconception to rest.

Autophagy researchers have no 'autophagometer' with which to measure autophagy or track its changes over time and under different conditions. New tools in this resurging field are bound to arrive. Yet with both new and old approaches, scientists will want to be sure to use multiple approaches to home in on this complex and dynamic process.

1. Klionsky, D. et al. Autophagy (in press).

2. Barth, S., Glick, D. \& Macleod, K.F. J. Pathol. 221, 117-124 (2010).

3. Sharifi, M. et al. Methods Mol. Biol. 1292 , 129-150 (2015).

4. Kogure, T. et al. Nat. Biotechnol. 24, 577-581 (2006).

5. Katayama, H. et al. Chem. Biol. 18, 1042-1052 (2011).

6. Lynch-Day, M.A. \& Klionsky, D.J. FEBS Lett. 584, 1359-1366 (2010).

Vivien Marx is technology editor for Nature and Nature Methods

(v.marx@us.nature.com). 